BMJ Open

Diabetes

Research

\& Care

\title{
Association of visceral adiposity and clinical outcome among patients with aldosterone producing adenoma
}

\author{
Leay Kiaw Er, ${ }^{1,2}$ Meng-Chun Lin, ${ }^{3}$ Yao-Chou Tsai, ${ }^{4,5}$ Jong-Kai Hsiao,,${ }^{6,7}$ \\ Chung-Yi Yang, ${ }^{8,9}$ Chin-Chen Chang, ${ }^{10,11}$ Kang-Yung Peng, ${ }^{11,12}$ Jeff S Chueh, ${ }^{13}$ \\ Vin-Cent Wu (D) , ${ }^{11,12}$ on behalf of the TAIPAI Study Group
}

To cite: Er LK, Lin M-C, Tsai Y-C, et al. Association of visceral adiposity and clinical outcome among patients with aldosterone producing adenoma. BMJ Open Diab Res Care 2020;8:e001153. doi:10.1136/ bmjdrc-2019-001153

- Additional material is published online only. To view please visit the journal online (http://dx.doi.org/10.1136/ bmjdrc-2019-001153).

Received 25 December 2019 Revised 11 April 2020 Accepted 10 June 2020
Check for updates

(c) Author(s) (or their employer(s)) 2020. Re-use permitted under CC BY-NC. No commercial re-use. See rights and permissions. Published by BMJ.

For numbered affiliations see end of article.

Correspondence to Dr Vin-Cent Wu; q91421028@ntu.edu.tw

\section{ABSTRACT}

Introduction Primary aldosteronism (PA) is a common form of secondary hypertension that has significant cardiovascular events and increased prevalence of metabolic syndrome and diabetics. Although plasma aldosterone concentration is positively correlated with visceral fat area (VFA) in non-PA individuals, the role of visceral adiposity associated with clinical success after surgery is not known.

Research design and methods We analyzed patients who underwent adrenalectomy for aldosterone-producing adenoma (APA) at the Taiwan PA Investigator group. VFA was calculated from the abdominal CT scan at APA diagnosis, and all patients received adrenalectomy. Results The study involved 100 consecutive patients with APA (42 males; mean age 49.3 years) matched with 41 essential hypertension (EH) patients. Patients with APA had smaller VFA $(p=0.010)$ than their EH counterparts. Multiple linear regression analysis revealed that the duration of hypertension ( $p=0.007$ ), but not plasma aldosterone, was negatively correlated with VFA in patients with APA. Logistic regression analysis showed that log VFA $(0 R=0.065, p<0.001)$ and duration of hypertension before PA diagnosis $(0 R=0.919, p=0.011)$ can predict complete clinical success after adrenalectomy. Multifactor-adjusted generalized additive model demonstrated that log VFA $<9.2$ was associated with complete cure of hypertension. Furthermore, VFA was increased at 6 months after adrenalectomy $(\mathrm{p}=0.045)$.

Conclusions Patients with APA had smaller VFA than their EH counterparts, and VFA increased after adrenalectomy. Clinical complete cure of hypertension after surgery was associated with smaller VFA and shorter duration of hypertension at PA diagnosis, suggesting a potential interplay of visceral adiposity and aldosterone of the patients with APA.

\section{INTRODUCTION}

Primary aldosteronism (PA), a state of aldosterone excess due to the autonomous production of aldosterone by adrenal adenoma or hyperplasia of the zona glomerulosa, affects $5 \%-13 \%$ of patients with hypertension. ${ }^{1}$ Patients with PA have an increased rate of cardiovascular events and renal damage, in addition to hypertension. ${ }^{23}$ Previous studies

\section{Significance of this study}

What is already known about this subject?

- Patients with primary aldosteronism $(\mathrm{PA})$ have an increased rate of cardiovascular events and renal damage, metabolic syndrome (MS) and diabetics in addition to hypertension.

- Aldosterone levels positively correlate with visceral adipose tissue area in healthy women, and 24-hours urinary aldosterone levels were also found to correlate with subcutaneous, not visceral, adipose tissue area in obese healthy individuals.

What are the new findings?

- Patients with PA has smaller visceral fat area (VFA) than their essential hypertension (EH) controls, and VFA increased after adrenalectomy.

- Smaller VFA and shorter duration of hypertensive latency could predict completely cure of hypertension after surgery.

How might these results change the focus of research or clinical practice?

- Chronic excess aldosterone will relate to decreased size of adipose tissue.

- VFA and duration of hypertension at diagnosis were related to long-term outcome after adrenalectomy. The complex interplay between aldosterone, adipose tissue, metabolic syndrome and subsequent diabetics in patients with PA needs further clarification.

showed impaired glucose homeostasis and insulin resistance (IR) in patients with PA, leading to increased prevalence of metabolic syndrome (MS),${ }^{4-6}$ which could be improved after adrenalectomy. ${ }^{47}$

Visceral adiposity, which is the accumulation of adipose tissue within the abdomen, is associated with cardiovascular disease MS and diabetics. ${ }^{8}$ Adipose tissue has been proposed to play a pivotal role in the pathogenesis of glucose metabolism disturbances in patients with PA. ${ }^{9}$ However, the gene expression of insulin signaling molecular from visceral adipose tissue is similar between APA and 
non-functioning adrenal adenoma, and thus the role of insulin sensitivity attributing to excess aldosterone is not conclusive in patients with aldosterone-producing adenoma (APA) ${ }^{10}$ However, aldosterone is an important contributor to adipogenesis via the non-genomic action of the mineralocorticoid receptor (MR) ${ }^{11}$ and evidence demonstrates that human adipocytes could also stimulate secretion of aldosterone and vice versa. ${ }^{12}{ }^{13}$ Aldosterone levels positively correlate with visceral adipose tissue area in healthy women, ${ }^{14}$ and 24-hour urinary aldosterone levels were also found to correlate with subcutaneous, not visceral, adipose tissue area in obese healthy individuals. ${ }^{15}$ However, the role of visceral adipose tissue and the outcome of patients with APA after surgery has not been well investigated. This study aimed to evaluate the role of visceral adiposity associated with clinical success after surgery in patients with APA.

\section{SUBJECTS AND METHODS}

Ethics statement

All participants signed the informed consent form before their enrollment in the research. The study performed all methods in agreement with approved guidelines.

\section{Patient selection and PA identification}

This study was conducted on patients with APA who received adrenalectomies from January 2011 to January 2013 and followed up until January 2014. The Taiwan primary aldosteronism investigators (TAIPAI) database was established for quality affirmation at two medical centers and their three affiliated hospitals and at two local hospitals from different cities in Taiwan. ${ }^{16-18}$ All antihypertensive medications were discontinued for at least 21 days before performing confirmatory tests. Diltiazem and/or doxazosin were administered to manage distinctly high blood pressure when needed. ${ }^{19} \mathrm{PA}$ was confirmed in patients with an elevated aldosterone after the saline infusion test, followed by imaging studies for subtype identification. PA confirmation and subtype identification were performed in hypertensive patients according to the standard protocol of the TAIPAI and aldosteronism consensus of Taiwan,,$^{20}$ including adrenal venous sampling and NP-59 scintigraphy with singlephoton emission computed tomography (SPECT-CT) imaging. ${ }^{18}$ Patients who were diagnosed with familiar hyperaldosteronism type I/Glucocorticoid Remediable Aldosteronism (GRA) were excluded using long-range PCR, as described previously. ${ }^{21}$

\section{Laboratory measurements}

We collected the baseline data of enrollees, including age, height, weight, waist circumference, blood pressure and biochemistry profile. Estimated glomerular filtration rate was ascertained by Modification of Diet in Renal Disease. ${ }^{22}$ Plasma aldosterone was measured by radioimmunoassay using a commercial kit (Aldosterone Maia Kit; Biochem Immunosystems, Bologna, Italy). The lowest detectable concentration of aldosterone was $10.0 \mathrm{pg} / \mathrm{mL}$, and the normal range of aldosterone was $70-350 \mathrm{pg} / \mathrm{mL}$ in the upright position. Plasma renin activity (PRA) was identified as the generation of angiotensin I by in vitro analysis using a commercially available radioimmunoassay kit (Stillwater, Minnesota, USA). The normal range of PRA was $2.63 \pm 1.32 \mathrm{ng} / \mathrm{mL} /$ hour in the upright position. The mean SD intra-assay and interassay coefficients of variation for the PRA assay were $1.9(5.0 \%)$ and 4.5 $(5.2 \%)$, respectively. The homeostasis model assessment of insulin resistance (HOMA-IR) was estimated using the following formula: HOMA-IR=HOMA-IR: insulin $(\mu \mathrm{U} /$ $\mathrm{mL}) \times$ glucose $(\mathrm{mg} / \mathrm{dL}) / 405 .^{23}$

\section{Measurement of abdominal adipose tissue by CT}

Imaging of each patient was performed with a 16-slice multidetector CT scanner (LightSpeed 16, GE Healthcare, Milwaukee, Wisconsin, USA). Image analysis software (ImageJ, V.1.43q; National Institutes of Health, Bethesda, Maryland, USA) was used for the subcutaneous and visceral abdominal adipose tissue areas at the level of the umbilicus, after applying threshold with an attenuation range between -50 to -250 Hounsfield units. The adipose tissue areas were presented in $\mathrm{mm} .{ }^{2}$ Subcutaneous fat area (SFA) was defined as the adipose tissue between the skin and muscle. Intra-abdominal tissue with density in the fat attenuation range was identified as visceral fat area (VFA) by CT as previously reported. ${ }^{24}$

\section{Adrenalectomy}

All of the operations were accomplished adopting the lateral transperitoneal approach and were executed by experienced laparoscopic surgeons to ensure that the principles of adrenal gland surgery were strictly followed. ${ }^{25}$

\section{Outcome measures}

Patients were followed up monthly for the first 3 months after the operation, and every 3 months from then on.

We defined the clinical outcome according to the Primary Aldosteronism Surgery Outcome consensus as complete clinical success indicating normal BP without antihypertensive medication at 1 year later after surgery. ${ }^{26}$

\section{Statistical analysis}

The correlations of VFA and SFA with plasma aldosterone, renin, aldosterone-renin ratio (ARR) and the other clinical parameters were examined using Pearson's correlation coefficients. The variables independently associated with VFA and SFA were identified by stepwise linear regression analysis. A normal distribution was attained by appropriate transformations of the skewed variables, including aldosterone, renin, ARR, VFA and SFA.

Binary logistic regression analysis with a stepwise variable selection procedure was adopted using available variables to identify the important factors associated with postoperative complete clinical success. The significance levels for entry and for stay were conservatively set at 0.15 . The goodness-of-fit (GOF) of the fitted multiple logistic regression model was examined by the estimated area 
Table 1 Characteristics of patients with APA and EH at diagnosis

\begin{tabular}{|c|c|c|c|}
\hline & EH & APA & \multirow[b]{2}{*}{$P$ value } \\
\hline & $(n=41)$ & $(n=100)$ & \\
\hline \multicolumn{4}{|l|}{ Male } \\
\hline Female (\%) & $24(58.5)$ & $58(58.0)$ & 0.999 \\
\hline Male(\%) & $17(41.5)$ & $42(42.0)$ & \\
\hline Age (years) & $53.1 \pm 12.4$ & $49.3 \pm 11.8$ & 0.090 \\
\hline Waist circumference (cm) & $84.1 \pm 11.6$ & $81.1 \pm 10.4$ & 0.167 \\
\hline BMI $\left(\mathrm{kg} / \mathrm{m}^{2}\right)$ & $24.9 \pm 3.6$ & $25.0 \pm 3.8$ & 0.919 \\
\hline $\mathrm{sBP}(\mathrm{mm} \mathrm{Hg})$ & $151.0 \pm 17.5$ & $154.1 \pm 22.9$ & 0.735 \\
\hline Log ald (ng/dL) & $3.4 \pm 0.7$ & $3.9 \pm 0.6$ & $<0.001$ \\
\hline Log renin (ng/mL/hour) & $-0.5 \pm 1.1$ & $-1.9 \pm 1.9$ & $<0.001$ \\
\hline $\begin{array}{l}\text { Log (ARR) } \\
\text { (ng/dL per ng/mL/hour) }\end{array}$ & $3.9 \pm 1.4$ & $5.8 \pm 2.0$ & $<0.001$ \\
\hline Potassium (mmol/L) & $4.2 \pm 0.4$ & $3.6 \pm 0.7$ & $<0.001$ \\
\hline Cholesterol (mg/dL) & $192.4 \pm 33.4$ & $190.2 \pm 34.0$ & 0.671 \\
\hline Triglyceride (mg/dL) & $108.4 \pm 58.1$ & $125.4 \pm 120.2$ & 0.938 \\
\hline HOMA-IR index & $3.2 \pm 6.0$ & $2.6 \pm 4.5$ & 0.152 \\
\hline eGFR $\left(\mathrm{mL} / \mathrm{min} / 1.73 \mathrm{~m}^{2}\right)$ & $90.3 \pm 28.0$ & $86.8 \pm 29.4$ & 0.409 \\
\hline Duration of HTN (years) & $5.8 \pm 6.3$ & $7.8 \pm 7.6$ & 0.100 \\
\hline $\log$ VFA $\left(\mathrm{mm}^{2}\right)$ & $9.3 \pm 0.5$ & $9.1 \pm 0.5$ & 0.021 \\
\hline $\log$ SFA $\left(\mathrm{mm}^{2}\right)$ & $9.8 \pm 0.4$ & $9.7 \pm 0.4$ & 0.117 \\
\hline
\end{tabular}

Data are expressed as mean \pm SD unless otherwise indicated. APA, aldosterone-producing adenoma; ARR, aldosteronerenin ratio; $\mathrm{BMI}$, body mass index; eGFR, estimate glomerular filtration rate; $\mathrm{EH}$, essential hypertension; HOMA-IR, homeostasis model assessment for insulin resistance; HTN, hypertension; sBP, systolic blood pressure; SFA, subcutaneous fat area; VFA, visceral fat area.

under the receiver operating characteristic curve, the adjusted generalized $\mathrm{R}^{2}$ and the Hosmer-Lemeshow GOF test. A generalized additive model (GAM) that incorporated the subject-specific random effects was plotted then adjusted for the duration of hypertension to diagnosis of $\mathrm{PA}^{.27-29}$

All continuous variables were reported as means \pm SDs (with 95\% CIs as appropriate), and categorical variables were reported as frequencies or percentages. A $p$ value of $<0.05$ was considered statistically significant. The statistical analyses were performed using SPSS software, V.22.0, and R software, V.2.8.1 (Free Software Foundation, Inc., Boston, Massachusetts).

\section{RESULTS}

\section{Patient characteristics}

During the study period, 100 APA patients (42 males; aged $49.3 \pm 11.8$ years) who had received adrenalectomy were identified (table 1). Furthermore, 41 patients with EH (17 males; 24 females) whose age, sex, body mass index and systolic blood pressure were matched and enrolled as a control group. The baseline characteristics before surgery were listed in table 1 .
Table 2 Correlations of VFA of APA patients with plasma aldosterone, renin, ARR and other clinical variables examined using Pearson's correlation coefficients

\begin{tabular}{|c|c|c|c|c|}
\hline & \multicolumn{2}{|c|}{ Univariate } & \multicolumn{2}{|c|}{ Multivariate } \\
\hline & $\mathbf{r}$ & $P$ value & Beta & $P$ value \\
\hline Age (years) & 0.278 & 0.001 & 0.015 & 0.001 \\
\hline BMI $\left(\mathrm{kg} / \mathrm{m}^{2}\right)$ & 0.522 & $<0.001$ & 0.056 & $<0.001$ \\
\hline Waist circumference $(\mathrm{cm})$ & 0.655 & $<0.001$ & 0.020 & $<0.001$ \\
\hline $\mathrm{sBP}(\mathrm{mm} \mathrm{Hg})$ & 0.024 & 0.775 & _ & - \\
\hline Log ald (ng/dL) & -0.058 & 0.492 & - & - \\
\hline Log renin (ng/mL/hour) & 0.167 & 0.048 & - & - \\
\hline $\begin{array}{l}\log (A R R) \\
\text { (ng/dL per ng/ml/hour) }\end{array}$ & -0.168 & 0.047 & - & - \\
\hline Potassium (mmol/L) & 0.216 & 0.010 & - & - \\
\hline Cholesterol (mg/dL) & 0.003 & 0.972 & - & - \\
\hline Triglyceride (mg/dL) & 0.193 & 0.022 & _ & - \\
\hline HOMA-IR index & 0.191 & 0.024 & - & - \\
\hline eGFR $\left(\mathrm{mL} / \mathrm{min} / 1.73 \mathrm{~m}^{2}\right)$ & -0.241 & 0.004 & -0.004 & 0.010 \\
\hline Duration of HTN (years) & 0.047 & 0.583 & -0.015 & 0.020 \\
\hline
\end{tabular}

Independent risks predicting preoperative VFA of APA patients by multiple linear regression model.

APA, aldosterone-producing adenoma; ARR, aldosteronerenin ratio; $\mathrm{BMI}$, body mass index; eGFR, estimate glomerular filtration rate; HOMA-IR, homeostasis model assessment for insulin resistance; HTN, hypertension; sBP, systolic blood pressure; VFA, visce fat area.

APA patients had smaller VFA $(\mathrm{p}=0.021)$, lower levels of renin $(\mathrm{p}<0.001)$ and potassium $(\mathrm{p}<0.001)$ and higher level of aldosterone $(\mathrm{p}<0.001)$ and ARR $(\mathrm{p}<0.001)$ than EH patents (table 1).

\section{Factors that correlated with VFA in patients with PA}

Multiple linear regression showed that age $(\mathrm{p}=0.001)$, BMI $(p<0.001)$, waist circumference $(b=0.020, p<0.001)$, and the duration of hypertension $(b=-0.015, p<0.001)$ were independent factors related to VFA in patients with APA (table 2). Furthermore, after adjusting the covariables, the non-linear GAM regression plot showed a trend for inverse association between log VFA and high 24-hour urine aldosterone level, especially when it was $>29.3 \mu \mathrm{g} /$ day (online supplementary figure $\mathrm{S} 1$ ).

\section{Factors that predicted cure of hypertension after adrenalectomy}

One year after adrenalectomy, 66 of 100 patients (66.0\%) were completely cured of hypertension (table 3 ).

The GAM regression model (figure 1) showed that APA patients with log VFA smaller than 9.2 had a clinical advantage in complete clinical success. Smaller VFA, identified as $\log$ VFA $<9.2$, has a sensitivity of $0.742(95 \%$ CI 0.620 to 0.842 ); specificity of 0.676 (95\% CI 0.495 to 0.826 ); positive predictive value of 0.817 (95\% CI 0.696 to 0.905$)$; and negative predictive value of $0.575(95 \%$ CI 0.409 to 0.730 ) to complete clinical success. Therefore, smaller Log VFA (OR=0.065; 95\% CI 0.018 to 0.231; 
Table 3 Characteristics of patients with APA at diagnosis categories by hypertensive status after adrenalectomy

\begin{tabular}{|c|c|c|c|c|}
\hline & $\begin{array}{l}\text { All patients } \\
(n=100)\end{array}$ & $\begin{array}{l}\text { Residual of hypertension } \\
(n=34)\end{array}$ & $\begin{array}{l}\text { Cure of hypertension } \\
(n=66)\end{array}$ & $P$ values \\
\hline Female (\%) & 58 (58.0\%) & 13 (38.2\%) & 45 (68.2\%) & 0.005 \\
\hline Male (\%) & $42(42.0 \%)$ & $21(61.8 \%)$ & $21(31.8 \%)$ & 0.005 \\
\hline Age (years) & $49.3 \pm 11.8$ & $53.3 \pm 12.4$ & $47.3 \pm 11.1$ & 0.016 \\
\hline Waist circumference (cm) & $81.1 \pm 10.4$ & $86.4 \pm 10.4$ & $78.4 \pm 9.4$ & $<0.001$ \\
\hline $\mathrm{BMI}\left(\mathrm{kg} / \mathrm{m}^{2}\right)$ & $25.0 \pm 3.8$ & $26.1 \pm 3.5$ & $24.5 \pm 3.8$ & 0.012 \\
\hline $\mathrm{sBP}(\mathrm{mm} \mathrm{Hg})$ & $154.1 \pm 22.9$ & $156.1 \pm 21.8$ & $153.1 \pm 23.5$ & 0.298 \\
\hline Log ald (ng/dL) & $3.9 \pm 0.6$ & $3.8 \pm 0.6$ & $4.0 \pm 0.6$ & 0.352 \\
\hline Log renin (ng/mL/hour) & $-1.9 \pm 1.9$ & $-1.9 \pm 2.1$ & $-1.9 \pm 1.8$ & 0.924 \\
\hline $\begin{array}{l}\text { Log (ARR) } \\
\text { (ng/dL per ng/mL/hour) }\end{array}$ & $5.8 \pm 2.0$ & $5.7 \pm 2.1$ & $5.9 \pm 2.0$ & 0.859 \\
\hline Potassium (mmol/L) & $3.6 \pm 0.7$ & $3.8 \pm 0.5$ & $3.5 \pm 0.7$ & 0.018 \\
\hline Cholesterol (mg/dL) & $190.2 \pm 34.0$ & $190.1 \pm 32.9$ & $190.3 \pm 34.9$ & 0.839 \\
\hline Triglyceride (mg/dL) & $125.4 \pm 120.2$ & $124.8 \pm 67.1$ & $125.7 \pm 140.4$ & 0.123 \\
\hline HOMA-IR index & $2.6 \pm 4.5$ & $3.2 \pm 5.7$ & $2.4 \pm 3.7$ & 0.184 \\
\hline eGFR $\left(\mathrm{mL} / \mathrm{min} / 1.73 \mathrm{~m}^{2}\right)$ & $86.8 \pm 29.4$ & $75.4 \pm 27.0$ & $92.6 \pm 29.0$ & 0.002 \\
\hline Duration of HTN (years) & $7.8 \pm 7.6$ & $10.4 \pm 7.6$ & $6.4 \pm 7.3$ & 0.004 \\
\hline Log VFA $\left(\mathrm{mm}^{2}\right)$ & $9.1 \pm 0.5$ & $9.4 \pm 0.4$ & $8.9 \pm 0.5$ & $<0.001$ \\
\hline $\log$ SFA $\left(\mathrm{mm}^{2}\right)$ & $9.7 \pm 0.4$ & $9.8 \pm 0.3$ & $9.6 \pm 0.4$ & 0.070 \\
\hline
\end{tabular}

Data are expressed as mean \pm SD unless otherwise indicated.

APA, aldosterone-producing adenoma; ARR, aldosterone-renin ratio; BMI, body mass index; eGFR, estimate glomerular filtration rate; HOMA-IR, homeostasis model assessment for insulin resistance; HTN, hypertension; sBP, systolic blood pressure; SFA, subcutaneous fat area; VFA, visceral fat area.

$\mathrm{p}<0.001)$ and shorter duration of hypertension to PA diagnosis $(\mathrm{OR}=0.919 ; 95 \%$ CI 0.860 to $0.981 ; \mathrm{p}=0.011)$ could independently predict the cure of hypertension. The model had well calibrated, Hosmer-Lemeshow GOF test $(\mathrm{p}=0.821)$. Because the possibility of interaction between BMI and VFA, six subgroups were constructed by stratified BMI into tertile values and combined with $\log$ VFA, which was either greater or smaller than 9.2 (online supplementary figure S2). We compared the ORs and $95 \%$ CIs of each subgroup for predicting the cure of hypertension. The size of VFA could predict the cure of hypertension, yet stratification into tertiles according to BMI could not.

\section{Change of VFA and SFA after adrenalectomy}

Twenty patients with APA (9 men, 11 women) underwent sequential CT at 6 months after adrenalectomy. The mean differences of log pre-adrenalectomy VFA versus $\log$ postadrenalectomy VFA was -0.069 (95\% CI -0.137 to 0.002 ) and log preadrenalectomy SFA versus log postadrenalectomy SFA was -0.059 (95\% CI -0.142 to 0.023$)$, respectively. The log VFA ( $\mathrm{p}=0.045)$ but not the log SFA $(\mathrm{p}=0.150)$ augmented after adrenalectomy (figure 2$)$.

\section{DISCUSSION}

The main finding of this multicenter study was that patients with PA had smaller VFA than their EH controls and that VFA increased after adrenalectomy. Smaller VFA and shorter duration of hypertensive latency could predict the complete cure of hypertension after surgery. Smaller VFA had a high discriminative power to complete clinical success with cure of hypertension. In this study, we showed that small VFA was associated with clinical complete success after surgery, even after adjustment for BMI. Previous study showed that PAC was positively correlated with visceral adipose tissue in non-PA population, ${ }^{14}$ and 24-hour urinary aldosterone excretion was also found positively correlated with SFA but not with the VFA of obese non-PA individuals. ${ }^{15}$ Our study further revealed that a relatively high urinary aldosterone level was correlated with smaller VFA in patients with APA.

\section{Comparison of VFA in patients with APA and EH}

There is a two-way interaction between adipose tissue and aldosterone. ${ }^{30}$ Previous evidence demonstrated that human adipocytes produce mineralocorticoid-releasing factors that stimulated secretion of aldosterone. ${ }^{12} 13$ Adipocytes may contribute to excess aldosterone synthesis and secretion, involving both generalized stimulation of the renin-angiotensin-aldosterone system and, independently, production and release of products that could stimulate local or adrenal aldosterone synthesis. ${ }^{31}$ In addition, aldosterone acts via non-genomic action on the MR and induces peroxisome proliferator activated 
VFA predicts cure of HTN after adrenalectomy

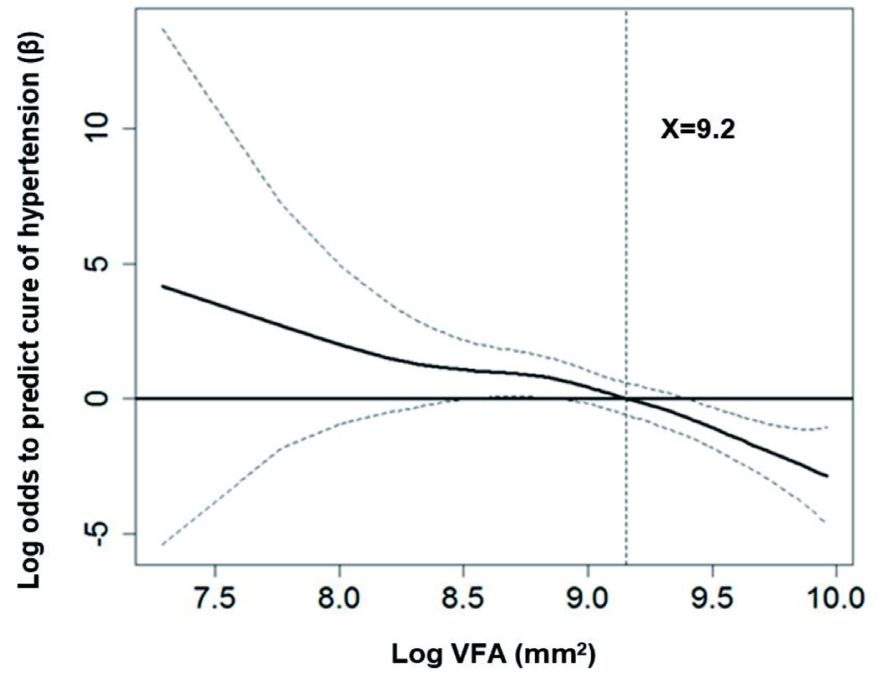

Figure 1 APA patients with log VFA smaller than 9.2 had a clinical advantage in clinical complete success. GAM plot for the probability of cure of hypertension against log VFA level of APA patients at index date incorporating the subject-specific random effects expressed as the logarithm of the odds (logit). The probability of outcome events was constructed with hypertensive duration have an average of zero over the range of the data, that is, log VFA=9.2. The dashed lines indicate approximated point-wise 95\% Cl. Data are expressed as mean $\pm S D$ unless otherwise indicated. APA, aldosterone-producing adenoma; GAM, generalized additive model; HTN, hypertension; VFA, visceral fat area.

receptor gamma mRNA expression, therefore promoting white adipose cell differentiation and leading to a proadipogenic effect on human adipocytes in vivo. ${ }^{113}$ However, evidence for the direct effect of chronic excess aldosterone among patients with $\mathrm{PA}$ on visceral adipose tissue is scarce. When adipose cells, 3T3-L1 and 3T3-F442A, were exposed to different doses of aldosterone, the proadipogenic effect of aldosterone was attenuated by higher doses of aldosterone. ${ }^{13}$ Additionally, a recent study that investigated perirenal adipose tissue, which is another region of visceral adipose tissue in the retroperitoneal

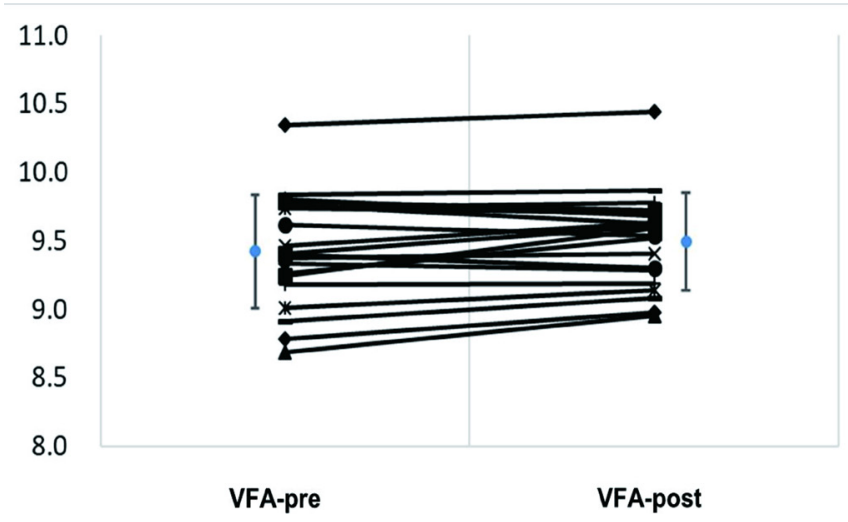

Figure 2 Log postoperation VFA (after adrenalectomy) and log preoperation VFA (before adrenalectomy) of 20 patients with APA. APA, aldosterone-producing adenoma; VFA, visceral fat area. cavity, and found interleukin 6 (IL-6), Tumor Necrosis Factor- $\alpha$ (TNF- $\alpha$ ) protein and mRNA were significantly higher in the APA group than in the Normotension (NT) and EH groups. ${ }^{32}$ The authors of that study proposed that excess aldosterone in vivo may be related to increased inflammation in perirenal adipose tissue. ${ }^{32}$

Importantly, the markers of fibrosis such as fibronectin and collagen I protein were reported to be higher in the perirenal adipose tissue in patients with APA than in NT and $\mathrm{EH}$ patients ${ }^{32}$ suggesting that aldosterone in patients with APA may cause perirenal adipose tissue impairment and lead to inflammation and fibrosis. Previous studies demonstrated an inverse correlation between fibrosis and adipocyte area. ${ }^{33}$ Taken together, it would be reasonable to speculate that hypersecretion of aldosterone increases tissue fibrosis and attenuates the size of adipocytes in vivo. Moreover, sympathetic overactivity was found in patients with $\mathrm{PA},{ }^{35}$ and it might contribute to more energy consumption as well as lead to decreasing size of adipose tissue. Based on the aforementioned finding, it may be plausible to assume that patients with $\mathrm{PA}$, with chronic excessive aldosterone, would have a smaller VFA compared with patients with EH as observed in our study.

\section{Smaller VFA correlated with longer duration of hypertension to the diagnosis in patients with PA}

Our results showed that the duration of hypertension was negatively correlated with VFA at PA diagnosis, and smaller VFA is correlated with longer duration of hypertension to diagnosis in patients with PA. Compared with non-PA obese population, patients with PA had chronic excessive exposure to autonomously produced aldosterone; this might have different effects on adipose tissue as their dosages are different from non-PA population. This speculation might explain why our result showed longer term of excess aldosterone in patients with APA presented with smaller VFA. Among the population of non-PA, one study found the positive correlation between serum aldosterone levels and visceral obesity in healthy women ${ }^{14}$; however, other studies from a large communitybased sample of non-PA patients ${ }^{36}$ and a recent study of APA patients, ${ }^{37}$ in agreement with our result, reveal that visceral adipose tissue was not associated with plasma aldosterone levels.

Adipose tissue, in particular visceral fat, secretes several adipokines that cause inflammation, energy balance and insulin resistant. ${ }^{38}$ This positive correlation between VFA and insulin resistance (HOMA-IR) in non-PA led to the detrimental effect on glucose and lipid metabolism. However, our investigation showed no correlation between VFA and HOMA-IR in patients with PA. We suggested that other factors might play more crucial roles than VFA as the causes of impaired glucose homeostasis and IR in patients with PA. Several factors have been proposed in previous studies. First, increased inflammation, oxidative stress, and a decreased beta cell function can be precipitated by increased aldosterone levels. ${ }^{39} 40$ 
Second, the increased aldosterone in PA caused IR in peripheral tissues. ${ }^{10} 4142$ Third, hypokalemia impaired insulin secretion. ${ }^{43-45}$

\section{Predictive factors for clinical complete success after adrenalectomy in patients with APA}

Our result showed that smaller VFA was a strong predictor of clinical complete success after adrenalectomy (table 3). It raises the possibility of using VFA and duration of hypertension to diagnosis PA as predictors in determining long-term outcome after adrenalectomy. Mounting evidence suggested that excess weight gain and visceral obesity were the major causes of hypertension. ${ }^{46}$ Thus, patients with APA who have large VFA might carry a concomitant obesity-related hypertension and have residual hypertension even after adrenalectomy. We suggested that APA patients with increased visceral adipose tissue following adrenalectomy should be evaluated and treated with raised awareness in order to achieve ideal glucose control and prevent potential complication.

Moreover, our study found shorter duration of hypertensive latency before PA diagnosis was a predictor of complete clinical cure of hypertension after adrenalectomy. In patients with PA, longer duration of hypertension is likely to be long-term exposure to excess aldosterone, and this phenomenon might contribute to endothelial dysfunction, vascular damage and arteriolosclerosis and may be related to cardiac remodeling and vascular hypertrophy. ${ }^{47}$ We speculated that patients with an APA as the sole cause of hypertension have factors that decrease VFA thus respond more completely to APA removal than patients who may have acquired an APA in addition to other pathologies causing hypertension.

Of note, CTs are readily obtained in almost every diagnosed patients with PA before lateralization. Compared with BMI, CT can quantify fat distribution and measure adiposity more accurately, ${ }^{48}$ while BMI cannot differentiate body fat from fat-free mass. Our subgroup analysis (online supplementary figure S2) demonstrated that the size of VFA, but not BMI, could predict the cure of hypertension even patients with APA were stratified in to tertiles according to BMI. Additionally, for those patients with APA who has lower BMI, APA patients with smaller VFA showed higher possibility for cure of hypertension after adrenalectomy. Finally, our study also revealed that VFA significantly increased after adrenalectomy. Similarly, Giacchetti et al ${ }^{49}$ reported that the body weight significantly increased in APA patients after adrenalectomy. This might lead to the attenuation of chronic excess aldosterone, which is related to decreased size of adipose tissue after adrenalectomy. ${ }^{33-35}$

\section{Study limitations}

Our study had the following limitations. First, our study was an observational study; therefore, the associations were not prospective, and causality cannot be inferred. The observational nature of this study was an intrinsic limitation because the lack of randomization precluded a definite investigation of treatment advantages. Observation data may currently remain the best available evidence to observe the association of PA with VFA. Moreover, this was a correlation study with no biological experimental evidence to validate the correlations or to state the role of aldosterone on MR of adipose tissue in patients with PA. Furthermore, as we did not perform $1 \mathrm{mg}$ Dexamethasone Suppression Test (DST) for all patients in this study, we could not completely exclude the possibility of the inclusion of patients with autonomous cosecretion of cortisol. One potential problem is the correlation between the cross-sectional areas of VFA in a single slice and the VFA volume quantified with multiple slices has different; nevertheless, strong correlations are developed regardless of sample size.$^{48}$ Future elegant research would need to focus on further delineation of the complex interplay between aldosterone and adipose tissue in a larger sample size of patients with PA, as well as to examine the correlation of plasma aldosterone levels and visceral adipose tissue in patients with PA.

\section{CONCLUSION}

In this study, we found a negative association between relatively high urine aldosterone level and VFA that was calculated from abdominal CT in patients with APA. Patients with APA had smaller VFA than EH patients and VFA could increase after surgery. Smaller VFA and shorter duration of hypertension could predict the complete clinical cure of hypertension after adrenalectomy. These results raised the possibility that VFA and duration of hypertension at diagnosis were related to long-term outcome after adrenalectomy. The complex interplay between aldosterone, adipose tissue and subsequent diabetics in patients with PA needs further clarification.

\section{Author affiliations}

${ }^{1}$ Division of Endocrinology and Metabolism, Department of Internal Medicine, Taipei Tzu-Chi Hospital, Buddhist Tzu-Chi Medical Foundation, New Taipei City, Taiwan

${ }^{2}$ School of Medicine, Tzu Chi University College of Medicine, Hualien, Taiwan

${ }^{3}$ Department of Internal Medicine, National Taiwan University Hospital, Chu-Tung Branch, Chu-Tung, Taiwan

${ }^{4}$ Department of Urology, Taipei Medical University Hospital, Taipei, Taiwan ${ }^{5}$ Department of Urology, School of Medicine, Taipei Medical University, Taipei, Taiwan

${ }^{6}$ College of Medicine, Tzu Chi University, Hualien, Taiwan

${ }^{7}$ Department of Radiology, Taipei Tzu-Chi Hospital, Buddhist Tzu-Chi Medical Foundation, New Taipei City, Taiwan

${ }^{8}$ Department of Radiology, E-Da Hospital, Kaohsiung City, Taiwan

${ }^{9}$ College of Medicine, I-Shou University, Kaohsiung City, Taiwan

${ }^{10}$ Department of Medical Imaging, National Taiwan University Hospital, Taipei, Taiwan

${ }^{11}$ College of Medicine, National Taiwan University, Taipei, Taiwan

${ }^{12}$ Department of Internal Medicine, National Taiwan University Hospital, Taipei, Taiwan

${ }^{13}$ Department of Urology, Cleveland Clinic, Cleveland, Ohio, USA

Collaborators TAIPAI Study Group: Tai-Shuan Lai, Shao-Yu Yang, Kao-Lang Liu, Chin-Chen Chang, Bo-Chiag Lee, Shuo-Meng Wang, Kuo-How Huang, Po-Chih Lin, Yen-Hung Lin, Lian-Yu Lin, Shih-Cheng Liao, Ruoh-Fang Yen, Ching-Chu 
Lu (National Taiwan University Hospital, Taipei, Taiwan), Chieh-Kai Chan (NTUH Hsin-Chu branch), Leay-Kiaw Er, Ya-Hui Hu, Chia-Hui Chang, Che-Hsiung Wu, YaoChou Tsai (Taipei Tzu Chi Hospital, Buddhist Tzu Chi Medical Foundation, Taipei, Taiwan), Shih-Chieh Jeff Chueh (Cleveland Clinic Institute of Urology and Kidneys), Chen-Hsun Ho (Taipei Medical University-Shuang Ho Hospital, Ministry of Health and Welfare), Wei-Chieh Huang (New Taipei City Hospital), Ying-Ying Chen (MacKay Memorial Hospital), Vin-Cent Wu (National Taiwan University Hospital,Taipei, Taiwan NTUH, Director of Coordinating Center).

Contributors LKE, V-CW, M-CL and JSC conceived and designed the study. LKE, Y$\mathrm{CT}, \mathrm{V}-\mathrm{CW}$ and $\mathrm{K}-\mathrm{YP}$ and collected the data. All authors contributed to data analysis and interpretation. LKE, M-CL and V-CW wrote the manuscript. All authors read and approved the final manuscript.

Funding This study was supported by grants from the Taiwan National Science Council (MOST 106-2314-B-002-166-MY3, MOST 107-2314-B-002-026-MY3), National Taiwan University Hospital-Taipei Veterans General Hospital Joint Research Program (NTUH-TVGH VN103-01, NTUH-TVGH VN104-07, NTUH-TVGH VN105-04) and National Taiwan University Hospital (105-S3061, 107-S3809, UN103-082, UN106-014, 105-P05, 106-P02, 107-T02).

Disclaimer Funding organizations had no role in any aspect of the research or manuscript preparation.

Competing interests None declared.

Patient consent for publication Not required.

Ethics approval The study obtained Ethical approval (approval number. 200611031R) from the institutional review board of the National Taiwan University Hospital, Taipei, Taiwan.

Provenance and peer review Not commissioned; externally peer reviewed.

Data availability statement Data are available on reasonable request. Data may be obtained from a third party and are not publicly available. Data are available from the TAIPAI study group. Information on how to access the data can be found on these contact details: VinCent Wu, MD, PhD. Phone: +886-2-23562082 Email: q91421028@ntu.edu.tw. ORCID ID: 0000-0001-7935-0991.

Open access This is an open access article distributed in accordance with the Creative Commons Attribution Non Commercial (CC BY-NC 4.0) license, which permits others to distribute, remix, adapt, build upon this work non-commercially, and license their derivative works on different terms, provided the original work is properly cited, appropriate credit is given, any changes made indicated, and the use is non-commercial. See: http://creativecommons.org/licenses/by-nc/4.0/.

ORCID iD

Vin-Cent Wu http://orcid.org/0000-0001-7935-0991

\section{REFERENCES}

1 Young WF. Minireview: primary aldosteronism--changing concepts in diagnosis and treatment. Endocrinology 2003;144:2208-13.

2 TAIPAI Study Group, Wu V-C, Chueh S-C, et al. Association of kidney function with residual hypertension after treatment of aldosterone-producing adenoma. Am J Kidney Dis 2009;54:665-73.

3 Milliez P, Girerd X, Plouin P-F, et al. Evidence for an increased rate of cardiovascular events in patients with primary aldosteronism. J Am Coll Cardiol 2005;45:1243-8.

4 Fallo F, Veglio F, Bertello C, et al. Prevalence and characteristics of the metabolic syndrome in primary aldosteronism. J Clin Endocrinol Metab 2006;91:454-9.

5 Zennaro M-C, Caprio M, Fève B. Mineralocorticoid receptors in the metabolic syndrome. Trends Endocrinol Metab 2009;20:444-51.

6 Catena C, Lapenna R, Baroselli S, et al. Insulin sensitivity in patients with primary aldosteronism: a follow-up study. J Clin Endocrinol Metab 2006;91:3457-63.

7 Wu V-C, Chueh S-CJ, Chen L, et al. Risk of new-onset diabetes mellitus in primary aldosteronism: a population study over 5 years. $J$ Hypertens 2017;35:1698-708.

8 Després J-P, Lemieux I, Bergeron J, et al. Abdominal obesity and the metabolic syndrome: contribution to global cardiometabolic risk. Arterioscler Thromb Vasc Biol 2008;28:1039-49.

9 Ronconi V, Turchi F, Appolloni G, et al. Aldosterone, mineralocorticoid receptor and the metabolic syndrome: role of the mineralocorticoid receptor antagonists. Curr Vasc Pharmacol 2012;10:238-46.
10 Urbanet R, Pilon C, Calcagno A, et al. Analysis of insulin sensitivity in adipose tissue of patients with primary aldosteronism. J Clin Endocrinol Metab 2010;95:4037-42.

11 Williams JS. Evolving research in nongenomic actions of aldosterone. Curr Opin Endocrinol Diabetes Obes 2013;20:198-203.

12 Guo C, Ricchiuti V, Lian BQ, et al. Mineralocorticoid receptor blockade reverses obesity-related changes in expression of adiponectin, peroxisome proliferator-activated receptor-gamma, and proinflammatory adipokines. Circulation 2008;117:2253-61.

13 Caprio M, Fève B, Claës A, et al. Pivotal role of the mineralocorticoid receptor in corticosteroid-induced adipogenesis. Faseb $\mathrm{J}$ 2007;21:2185-94.

14 Goodfriend TL, Kelley DE, Goodpaster BH, et al. Visceral obesity and insulin resistance are associated with plasma aldosterone levels in women. Obes Res 1999;7:355-62.

15 Harada E, Mizuno Y, Katoh D, et al. Increased urinary aldosterone excretion is associated with subcutaneous not visceral, adipose tissue area in obese individuals: a possible manifestation of dysfunctional subcutaneous adipose tissue. Clin Endocrinol 2013;79:510-6.

16 Wu V-C, Chang H-W, Liu K-L, et al. Primary aldosteronism: diagnostic accuracy of the losartan and captopril tests. Am J Hypertens 2009;22:821-7.

17 Kuo C-C, Wu V-C, Huang K-H, et al. Verification and evaluation of aldosteronism demographics in the Taiwan primary aldosteronism investigation group (TAIPAl group). J Renin Angiotensin Aldosterone Syst 2011;12:348-57.

18 Chao C-T, Wu V-C, Kuo C-C, et al. Diagnosis and management of primary aldosteronism: an updated review. Ann Med 2013;45:375-83.

19 Wu KD, Liao TS, Chen YM, et al. Preoperative diagnosis and localization of aldosterone-producing adenoma by adrenal venous sampling after administration of metoclopramide. J Formos Med Assoc 2001;100:598-603.

20 Wu V-C, Hu Y-H, Er LK, et al. Case detection and diagnosis of primary aldosteronism - The consensus of Taiwan Society of Aldosteronism. J Formos Med Assoc 2017;116:993-1005.

21 Mulatero P, Curnow KM, Aupetit-Faisant B, et al. Recombinant CYP11B genes encode enzymes that can catalyze conversion of 11-deoxycortisol to cortisol, 18-hydroxycortisol, and 18-oxocortisol. $J$ Clin Endocrinol Metab 1998;83:3996-4001.

22 Levey AS, Bosch JP, Lewis JB, et al. A more accurate method to estimate glomerular filtration rate from serum creatinine: a new prediction equation. Modification of diet in renal disease Study Group. Ann Intern Med 1999;130:461-70.

23 Gastaldelli A, Miyazaki Y, Pettiti M, et al. Metabolic effects of visceral fat accumulation in type 2 diabetes. J Clin Endocrinol Metab 2002;87:5098-103

24 Wu C-K, Yang C-Y, Lin J-W, et al. The relationship among central obesity, systemic inflammation, and left ventricular diastolic dysfunction as determined by structural equation modeling. Obesity 2012:20:730-7.

25 Chen S-F, Chueh S-C, Wang S-M, et al. Clinical outcomes in patients undergoing laparoscopic adrenalectomy for unilateral aldosterone producing adenoma: partial versus total adrenalectomy. $J$ Endourol 2014;28:1103-6.

26 Williams TA, Lenders JWM, Mulatero P, et al. Outcomes after adrenalectomy for unilateral primary aldosteronism: an international consensus on outcome measures and analysis of remission rates in an international cohort. Lancet Diabetes Endocrinol 2017;5:689-99.

27 Shu K-H, Wang C-H, Wu C-H, et al. Urinary $\pi$-glutathione Stransferase predicts advanced acute kidney injury following cardiovascular surgery. Sci Rep 2016;6:26335.

28 Woo M-J, Reiter JP, Karr AF. Estimation of propensity scores using generalized additive models. Stat Med 2008;27:3805-16.

29 Wu V-C, Lo S-C, Chen Y-L, et al. Endothelial progenitor cells in primary aldosteronism: a biomarker of severity for aldosterone vasculopathy and prognosis. J Clin Endocrinol Metab 2011;96:3175-83.

30 Ronconi V, Turchi F, Bujalska IJ, et al. Adipose cell-adrena interactions: current knowledge and future perspectives. Trends Endocrinol Metab 2008;19:100-3.

31 Gorzelniak K, Engeli S, Janke J, et al. Hormonal regulation of the human adipose-tissue renin-angiotensin system: relationship to obesity and hypertension. J Hypertens 2002;20:965-73.

32 Wu C, Zhang $\mathrm{H}$, Zhang J, et al. Inflammation and fibrosis in perirenal adipose tissue of patients with aldosterone-producing adenoma. Endocrinology 2018;159:227-37.

33 Dankel SN, Svärd J, Matthä S, et al. COL6A3 expression in adipocytes associates with insulin resistance and depends on PPAR $\gamma$ and adipocyte size. Obesity 2014;22:1807-13. 
34 Divoux A, Tordjman J, Lacasa D, et al. Fibrosis in human adipose tissue: composition, distribution, and link with lipid metabolism and fat mass loss. Diabetes 2010;59:2817-25.

35 Kontak AC, Wang Z, Arbique D, et al. Reversible sympathetic overactivity in hypertensive patients with primary aldosteronism. $J$ Clin Endocrinol Metab 2010;95:4756-61.

36 O'Seaghdha CM, Hwang S-J, Vasan RS, et al. Correlation of renin angiotensin and aldosterone system activity with subcutaneous and visceral adiposity: the Framingham heart study. BMC Endocr Disord 2012;12:3.

37 Shibayama Y, Wada N, Baba S, et al. Relationship between visceral fat and plasma aldosterone concentration in patients with primary aldosteronism. J Endocr Soc 2018;2:1236-45.

38 Vettor R, Milan G, Rossato M, et al. Review article: adipocytokines and insulin resistance. Aliment Pharmacol Ther 2005;22(Suppl 2):3-10.

39 Jin HM, Zhou DC, Gu HF, et al. Antioxidant N-acetylcysteine protects pancreatic $\beta$-cells against aldosterone-induced oxidative stress and apoptosis in female $\mathrm{db} / \mathrm{db}$ mice and insulin-producing MIN6 cells. Endocrinology 2013;154:4068-77.

40 Mosso LM, Carvajal CA, Maiz A, et al. A possible association between primary aldosteronism and a lower beta-cell function. J Hypertens 2007;25:2125-30.

41 Fischer E, Adolf C, Pallauf A, et al. Aldosterone excess impairs first phase insulin secretion in primary aldosteronism. J Clin Endocrinol Metab 2013;98:2513-20.
42 Sherajee SJ, Fujita Y, Rafiq K, et al. Aldosterone induces vascular insulin resistance by increasing insulin-like growth factor-1 receptor and hybrid receptor. Arterioscler Thromb Vasc Biol 2012;32:257-63.

43 Corry DB, Tuck ML. The effect of aldosterone on glucose metabolism. Curr Hypertens Rep 2003;5:106-9.

44 Fallo F, Federspil G, Veglio F, et al. The metabolic syndrome in primary aldosteronism. Curr Diab Rep 2008;8:42-7.

45 Hanslik G, Wallaschofski H, Dietz A, et al. Increased prevalence of diabetes mellitus and the metabolic syndrome in patients with primary aldosteronism of the German Conn's registry. Eur $J$ Endocrinol 2015;173:665-75.

46 Hall JE, do Carmo JM, da Silva AA, et al. Obesity-induced hypertension: interaction of neurohumoral and renal mechanisms. Circ Res 2015;116:991-1006.

47 Pitt B, Zannad F, Remme WJ, et al. The effect of spironolactone on morbidity and mortality in patients with severe heart failure. randomized Aldactone evaluation study Investigators. N Engl J Med 1999;341:709-17.

48 Shuster A, Patlas M, Pinthus $\mathrm{JH}$, et al. The clinical importance of visceral adiposity: a critical review of methods for visceral adipose tissue analysis. Br J Radiol 2012;85:1-10.

49 Giacchetti G, Ronconi V, Turchi F, et al. Aldosterone as a key mediator of the cardiometabolic syndrome in primary aldosteronism: an observational study. J Hypertens 2007;25:177-86. 